\title{
Structural study of pure silica and Ge- containing zeolite ITQ-24
}

\author{
J.L. Jordá*, A. Cantín, A. Corma, M.J. Diaz-Cabañas, \\ S. Leiva, M. Moliner, F. Rey, M.J. Sabater, S. Valencia
}

Instituto de Tecnología Química (CSIC-UPV). Av. de los Naranjos s/n. 46022 Valencia (Spain)

*Contact author; e-mail: jjorda@itq.upv.es

Keywords: powder diffraction, zeolite, negative thermal expansion, germanium, EPDIC-10

\begin{abstract}
The possibility to obtain zeolite ITQ-24 in a wide Si/Ge range of compositions have made possible to study the preferential location of Ge atoms in the zeolitic framework. Also, it has allowed studying the effect of zeolite composition on the thermal properties of these materials, varying their composition while maintaining a neutral framework without extra-framework cations.
\end{abstract}

\section{Introduction}

Zeolites are microporous materials of great interest not only for academia, but also for their potential application as adsorbents, ion exchangers and catalysts; also, recently, some applications have been described for medicine, optics, magnetism and nanotechnology [1].

The main properties and applications of zeolites are closely related not only to their chemical composition, but also to the size and spatial distribution of the channels and cavities of their porous structures. Up to now, 167 different structures have been accepted by the International Zeolite Association [2].

Zeolite ITQ-24 (IWR) possesses interconnected 10 and 12 ring pores, and represents one end member of the CON family, formed also by SSZ-26, SSZ-33 and CIT-1 [3-5]; however, differently to the other members, ITQ-24 presents double four ring units (D4R) in its structure. Then, it is possible to synthesize ITQ-24 as a germanosilicate by taking advantage of the fact that the incorporation of Ge during the synthesis generally promotes the formation of zeolitic structures containing D4R units [6].

More recently, the combination of rational design and high-throughput techniques has allowed us to obtain this zeolite as pure-silica form [7]. It is notorious that the combination of X-ray powder diffraction and solid state ${ }^{29} \mathrm{Si}$ NMR of the calcined pure silica sample allowed to determine that the real symmetry of this material (space group Amm2) is slightly lower than that originally proposed $(\mathrm{Cmmm})$, with twice number of symmetry-independent $\mathrm{T}$-sites. Also, it has been described previously that many zeolites present an unusual negative thermal expansion [8-12]. However, all the results obtained were referred exclusively to pure silica 
and to aluminium-containing zeolites, where the presence of charge-compensating cations could largely affect the thermal behaviour of the framework.

Now, the possibility to obtain highly crystalline pure ITQ-24 zeolites within a wide range of $\mathrm{Si} / \mathrm{Ge}$ compositions, keeping in all cases framework electro-neutrality, allows performing the study of the effect of the framework composition on the thermal expansion of these materials, avoiding the influence of extra-framework species.

\section{Sample preparation and data collection}

Pure silica and germanium containing ITQ-24 samples were prepared as previously reported [7]. The samples were calcined in situ at $700^{\circ} \mathrm{C}$ under a dry $\mathrm{He} / \mathrm{O}_{2}$ atmosphere $(75 / 25$ volume $\%$ ) in an XRK-900 reaction chamber of Anton-Paar, attached to a PANalytical X'Pert PRO diffractometer, to remove the occluded organic. After cooling at room temperature, the X-ray diffraction pattern of each sample was collected in Bragg-Brentano geometry using an $\mathrm{X}$ 'Celerator detector and $\mathrm{Cu} \mathrm{K} \alpha_{1,2}$ radiation (tube voltage and intensity: $45 \mathrm{kV}$ and $40 \mathrm{~mA}$; fixed divergence slit $\left(1 / 16^{\circ}\right)$; scan range: $3-75^{\circ} 2 \theta$; step size: $0.017^{\circ} 2 \theta$, counting time: 1000 $\mathrm{s} /$ step). Then, subsequent patterns were collected at increasing temperatures using the same conditions as at the measurements performed at room temperature, with a counting time of $200 \mathrm{~s} / \mathrm{step}$ (heating rate: $4 \% \mathrm{~min}$; stabilization time before each measurement: $30 \mathrm{~min}$ ).

Rietveld refinements of the structures with different germanium contents were performed with the program FULLPROF [13] using the data collected at room temperature. The distribution of $\mathrm{Ge}$ in the different $\mathrm{T}$-sites was calculated from the different scattering factors of $\mathrm{Si}$ and Ge. Although the structures were refined in the Amm2 group, the occupancies of the Tsites that were symmetry-related in $\mathrm{Cmmm}$ have been constrained to be equal for stability of the refinements.

The cell parameters of the zeolites at different temperatures were calculated from the profile refinement of the diffraction patterns using FULLPROF.

\section{Results and discussion}

\section{Distribution of germanium in the framework}

Due to the larger ionic radius of $\mathrm{Ge}$ compared with $\mathrm{Si}$, the incorporation of Ge produces an increase of the cell parameters and the cell volume (Table 1), confirming that it is replacing Si isomorphically in the ITQ-24 framework.

Table 1. Unit cell parameters for pure silica and Ge-containing zeolite ITQ-24.

\begin{tabular}{|c|l|l|l|l|}
\hline Ge/unit cell & $a$-axis $(\AA)$ & $b$-axis $(\AA)$ & $c$-axis $(\AA)$ & Volume $\left(\AA^{3}\right)$ \\
\hline 0,0 & $12,4816(5)$ & $21,0634(9)$ & $13,4590(5)$ & $3538.4(2)$ \\
\hline 2,9 & $12,5097(11)$ & $21,1605(19)$ & $13,4690(11)$ & $3565.4(5)$ \\
\hline 4,4 & $12,5538(13)$ & $21,2101(25)$ & $13,4975(15)$ & $3593.9(7)$ \\
\hline 8,1 & $12,5828(13)$ & $21,2594(23)$ & $13,5163(11)$ & $3615.6(6)$ \\
\hline 8,6 & $12,5928(14)$ & $21,2661(25)$ & $13,5317(16)$ & $3623.8(7)$ \\
\hline 12,6 & $12,6478(12)$ & $21,3451(18)$ & $13,5727(10)$ & $3664.2(5)$ \\
\hline 15,7 & $12,6591(6)$ & $21,3646(10)$ & $13,5857(6)$ & $3674.3(3)$ \\
\hline
\end{tabular}


The Rietveld refinement of the data collected at room temperature for the calcined samples allows determining the distribution of Ge among the different T-sites.

As shown in figure 1, Ge locates preferentially at T1, corresponding to the double 4-rings (D4R) in the structure. This effect seems to be a general trend in Ge-containing zeolites, as previously found for other structures as ITQ-17 [14], ITQ-21 [15] or ITQ-22 [16]. It is particularly interesting that there exists an almost linear correlation between the number of Ge atoms in the unit cell and in the $\mathrm{T} 1$ sites along the complete range of $\mathrm{Si} / \mathrm{Ge}$ ratios studied in this work.

Ge occupation in T2 and T3 follows a similar tendency, being the replacement in T3 always higher than in T2. In both T-sites, Ge content slowly increases with the total Ge content in the unit cell up to a value of $12 \mathrm{Ge}$ per cell, which corresponds to a $50 \%$ replacement of $\mathrm{Si}$ with Ge in the T1 sites. When this value is reached, the Ge occupancy in the T2 and T3 sites rises faster, as previously reported for ITQ-17 [14].

Last, the case of T4, corresponding to the single 4-ring is anomalous. In this case, T4 is the second preferred site for Ge location, after T1, up to a value around 8 Ge per unit cell. Above this value, however, the occupation clearly decreases, been the less preferred site in the material containing 15.7 Ge per unit cell.

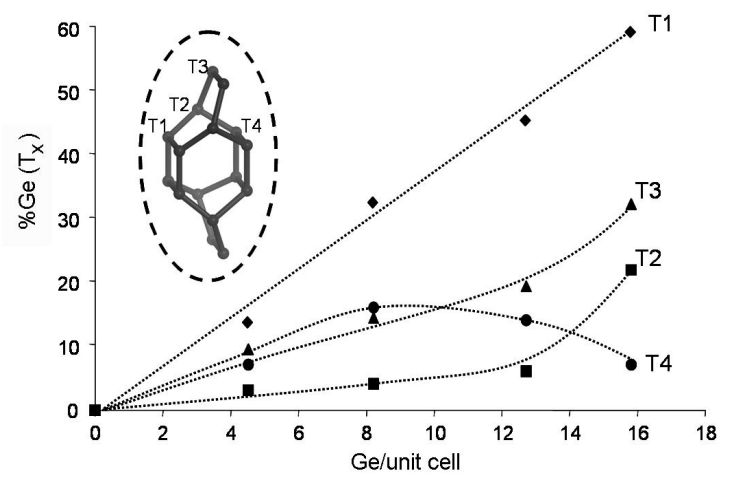

Figure 1. Distribution of Ge among the different T-sites in ITQ-24 samples as a function of the Ge content. The inset indicates the location of the T-sites in the structure

Although the X-ray diffraction data gives information on the overall substitution of Ge atoms at $\mathrm{T} 1$ sites, no information about the distribution of these atoms among the D4R cages is provided.

In order to elucidate the real composition of these cages, the use of additional ${ }^{19} \mathrm{~F}$ MAS NMR data proves to be extremely helpful. Due to the fact that fluorine atoms tend to be located inside the D4R units, and taking into account that this technique is very sensitive to the chemical environment of fluorine, it can provide evidence for the possible heterogeneity in the D4R composition. [12, 17]

Figure 2 shows the ${ }^{19} \mathrm{~F}$ MAS NMR spectra of five ITQ-24 non-calcined samples with different amounts of Ge per unit cell. The spectrum of the Ge-free sample shows only one band at 
$-38 \mathrm{ppm}$, corresponding to fluoride anions located in pure silica D4R cages. The incorporation of Ge atoms entails the apparition of two new bands at -19 and $-8 \mathrm{ppm}$, assigned to fluoride inside cages with 1 and 4 Ge respectively. The broadening of the latter band suggests either the presence of a certain disorder in the cages or the overlapping of additional bands corresponding to 3 or $5 \mathrm{Ge}$ atoms per cage. As the Ge content increases, a progressive decrease in the band at $-38 \mathrm{ppm}$ first, and in the band at $-19 \mathrm{ppm}$ for higher amounts of $\mathrm{Ge}$, is observed. This reduction in accompanied by an increase in the band at $-8 \mathrm{ppm}$, which is the unique band observable for the sample containing $15.7 \mathrm{Ge}$ per unit cell. The slight displacement of this band towards $-7 \mathrm{ppm}$ and the fact that, according to the XRD data, the Ge substitution in the D4R can reach values higher than 50\% indicates that, probably, the band broadening is mainly due to the contribution of, at least, one additional band corresponding to Ge-richer cages.

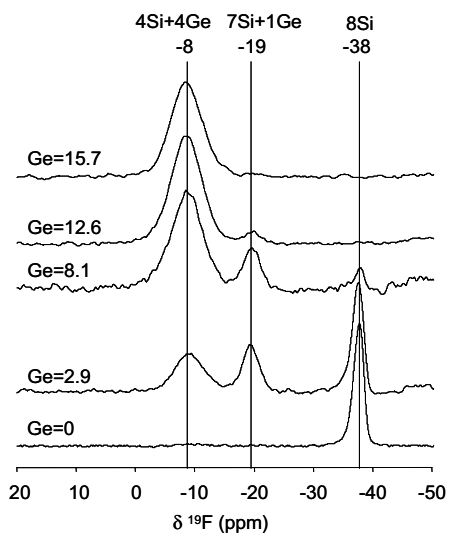

Figure 2. ${ }^{19}$ F MAS NMR spectra of the as-prepared ITQ-24 samples with different Ge contents.

\section{Thermal expansion of ITQ-24}

As can be seen in figure 3, the thermal behaviour of zeolite ITQ-24 is extremely dependent on its chemical composition.

Pure silica ITQ-24 presents a negative thermal expansion, typical in zeolites, along the $a$ and $c$ axis. However, this material presents an unusual positive expansion at temperatures below $200^{\circ} \mathrm{C}$ along the $b$ axis; above this temperature, the tendency changes and exhibits a negative expansion. In contrast, the $a$ parameter of the Ge-richest sample ( $15.7 \mathrm{Ge}$ per unit cell) is almost insensible to temperature, while $c$ decreases very slightly and $b$ clearly diminishes when increasing temperature. In both materials, the behaviour along $a$ and $c$ is similar and very different along $b$, the direction containing the higher density of 4R and D4R units; however, their cell volumes change almost identically.

For compositions in between, it is clear that the thermal behaviours are not linearly related with their compositions. Therefore, the cell of the sample containing 8.1 Ge presents a relative variation of the $a$ and $c$ parameters almost identical that for the pure silica sample, but $b$ coincides with the Ge-richest sample. Finally, the sample with 12.6 Ge per unit cell exhibits 
a very different behaviour; up to $300^{\circ} \mathrm{C}$ it presents the largest negative expansion but, above this temperature, the cell parameters remain constant in the temperature range studied.
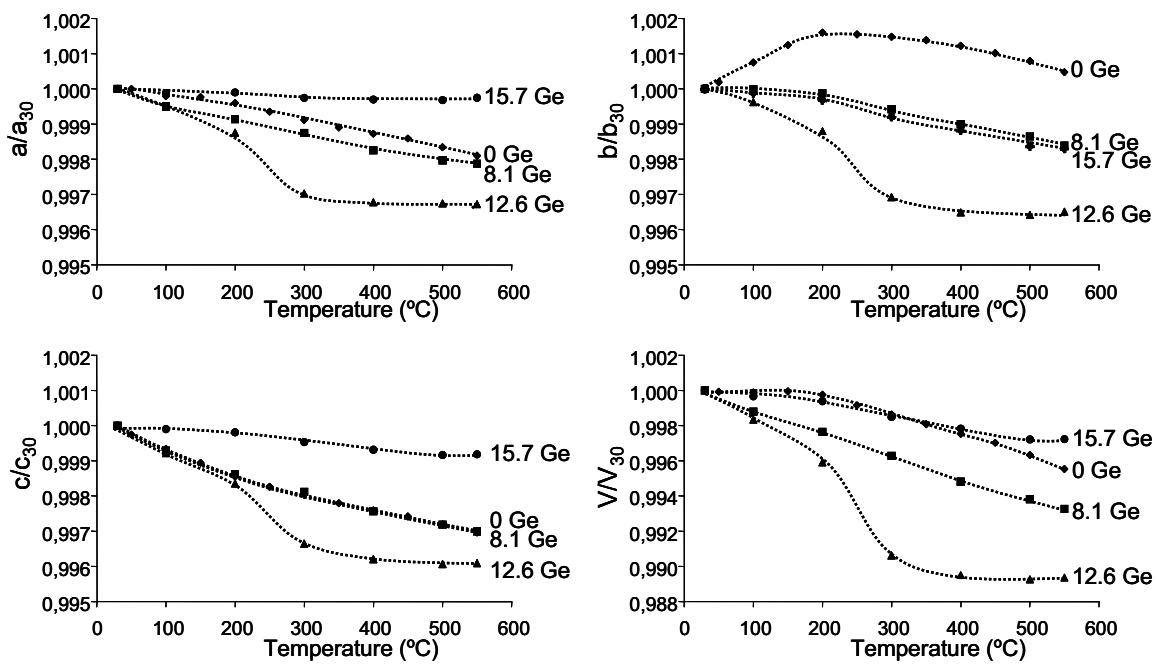

Figure 3. Variation of the unit cell parameters with temperature for ITQ-24 samples with four different germanium contents. All the parameters have been normalized respect to the values at $30^{\circ} \mathrm{C}$.

\section{Concluding remarks}

The possibility of synthesizing the zeolite ITQ-24 in a large range of $\mathrm{Si} / \mathrm{Ge}$ compositions allows studying the effect of Ge content on preferential occupation and thermal behaviour. Ge atoms tend to locate in the D4R, which seems to be a general trend for Ge-containing zeolites; once reached a 50\% occupancy in those cages, occupancy of T2 and T3 starts to rise faster. Occupancy of T4 in ITQ-24 is unusual, increasing up to $8 \mathrm{Ge}$ per cell, and decreasing after this value. Composition plays a critical role on the thermal behaviour of ITQ-24. Depending on the Ge content and on the working temperature range, the thermal expansion of this zeolite, clearly anisotropic along the $b$ axis, can be positive, negative or even zero, yielding interesting properties to this material.

\section{References}

1. Davis, M. E., 2002, Nature, 417, 813.

2. http://www.iza-structure.org/databases/

3. Lobo, R. F., Pan, M., Chan, I., Li, H. X.; Medrud, R. C., Zones, S. I., Crozier, P. A. \& Davis, M. E., 1993, Science, 262, 1543.

4. Zones, S. I., Olmstead, M. M. \& Santilli, D. S., 1992, J. Am. Chem. Soc.,114, 4195.

5. Lobo, R. F. \& Davis, M. E., 1995, J. Am. Chem. Soc., 117, 3766. 
6. Castañeda, R., Corma, A., Fornes, V., Rey, F. \& Rius, J., 2003, J. Am. Chem. Soc., 125, 7820 .

7. Cantín, A., Corma, A., Diaz-Cabañas, M.J., Jordá, J. L. \& Moliner, M., 2006, J. Am. Chem. Soc., 128, 4216.

8. Lightfoot, P., Woodcock, D. A., Maple, M. J., Villaescusa, L. A. \& Wright, P. A., 2001, J. Mater. Chem., 11, 212.

9. Martínez-Iñesta, M. M., \& Lobo, R. F., 2005, J. Phys. Chem. B, 109, 9389.

10. Park, S.H., Kunstleve, R. W. G., Graetsch, H. \& Gies, H, 1997, Prog. Zeo. Micropor. Mater., Part A-C, 105, 1989.

11. Bull, I., Lightfoot, P., Villaescusa, L. A., Bull, L. M., Gover R. K. B., Evans J. S. O. \& Morris, R. E., 2003, J. Am. Chem. Soc., 125, 4342

12. Villaescusa, L. A., Lightfoot, P., Teat, S. J. \& Morris, R. E., 2001, J. Am. Chem. Soc., 123, 5453

13. Rodriguez-Carvajal, J. FULLPROF version 3.30 (2005), LLB (unpublished).

14. Sastre, G., Vidal-Moya, J. A., Blasco, T., Rius, J., Jordá, J. L., Navarro, M. T., Rey, F. \& Corma, A, 2002, Angew. Chem. Int. Ed., 41, 4722.

15. Corma, A., Díaz-Cabañas, M. J., Martínez-Triguero, J., Rey, F., \& Rius, J., 2002, Nature, 418, 514.

16. Corma, A., Rey, F., Valencia, S., Jordá, J. L. \& Rius, J., 2003, Nature Materials, 2 , 493.

17. Caullet, P., Guth, J.L., Hazm, J., Lamblin, J.M. \& Gies, H., 1991, Eur. J. Solid State Inorg. Chem., 28, 345

Acknowledgements. J.L.J thanks the Spanish MEC for a Juan de la Cierva contract, and M.M. to CSIC for an I3P grant. Authors thank Dr. Alejandro Vidal-Moya and Estrella Mateos for NMR measurements. The authors thank the Spanish CICYT (MAT2003-07945C02-01 and MAT2003-07769-C02-01) and Generalitat Valenciana (project GV04B-270) for financial support. 